\title{
Developmental Human-Robot Imitation Learning of Drawing with a Neuro Dynamical System
}

\author{
Keita Mochizuki*, Shun Nishide ${ }^{\dagger}$, Hiroshi G. Okuno*, and Tetsuya Ogata ${ }^{\ddagger}$ \\ ${ }^{*}$ Graduate School of Infomatics, Kyoto University, Kyoto, Japan 606-8501 \\ Email: \{motizuki, okuno\}@kuis.kyoto-u.ac.jp \\ † the Hakubi Center for Advanced Research, Kyoto University, Kyoto, Japan 606-8501 \\ Email: nishide@kuis.kyoto-u.ac.jp \\ $\ddagger$ School of Fundamental Science and Engineering, Waseda University, Tokyo, Japan 169-8555 \\ Email: ogata@waseda.jp
}

\begin{abstract}
This paper mainly deals with robot developmental learning on drawing and discusses the influences of physical embodiment to the task. Humans are said to develop their drawing skills through five phases: 1) Scribbling, 2) Fortuitous Realism, 3) Failed Realism, 4) Intellectual Realism, 5) Visual Realism. We implement phases 1) and 3) into the humanoid robot NAO, holding a pen, using a neuro dynamical model, namely Multiple Timescales Recurrent Neural Network (MTRNN). For phase 1), we used random arm motion of the robot as body babbling to associate motor dynamics with pen position dynamics. For phase 3), we developed incremental imitation learning to imitate and develop the robot's drawing skill using basic shapes: circle, triangle, and rectangle. We confirmed two notable features from the experiment. First, the drawing was better performed for shapes requiring arm motions used in babbling. Second, performance of clockwise drawing of circle was good from beginning, which is a similar phenomenon that can be observed in human development. The results imply the capability of the model to create a developmental robot relating to human development.
\end{abstract}

Index Terms-Cognitive Developmental Robotics, Imitation Learning, Body Babbling, Neuro Dynamical System

\section{INTRODUCTION}

Cognitive developmental robotics is one of the attractive fields integrating research in robotics, cognitive science, and developmental science [1]. Works in this field have the following two goals.

1) constructivist goal : implementation of human cognitionbased models into robot to contribute to human understanding through robot analysis.

2) engineering goal: creation of a developmental robot.

One of the key concepts on cognitive developmental robotics is "physical embodiment", an effect or a property to learning provided through interaction between a physical body and environment such as objects or human. In this approach, a system is constructed with few preliminary manual design for the robot to autonomously learn by self-experience of interaction with environment.

In this paper, we focus on physical embodiment of drawing based on human infant's cognition of drawing skill. Drawing is a good example which greatly involves physical embodiment while easy to analyze.

The process of human development on drawing is defined with the following five phase by Louquet [2].

1) Scribbling (1-3 yrs)

2) Fortuitous Realism (2-4 yrs)

3) Failed Realism (3-7 yrs)

4) Intellectual Realism (4-8 yrs)

5) Visual Realism (8+ yrs)

1) is a phase to randomly move the arm to draw something of little significance. An infant starts to learn the relationship between the sense of his body through drawing experience with a pen in this phase. 2) is a phase to start having an intention to capy by discovering similarity between something drawn and objects existing in the real world and start having an intention to copy. 3) is a phase that an infant tries to copy objects he looks at but cannot perfectly copy it due to shortage of physical capability to draw. 4) is a phase to draw imagined virtual objects. Finally, 5) is a phase to draw perfectly by copying something the infant looks at. In our study, we focus on phases 1) and 3) as 4) and 5) have many factors that are complex to reproduce without the success of 1) to 3). Phase 2) is omitted since the phase involves relating drawn objects with the real world. In this paper, we focus only on the drawing mechanism of the robot.

We utilize the MTRNN (Multiple Timescales Recurrent Neural Network), proposed by Tani et al. [3], as the model of the robot. As the MTRNN is a neuro dynamical model, it is suitable for discussing physical embodiment involving dynamics. In addition, we can comprehend how the capability of drawing is acquired by analyzing the internal structure of the trained MTRNN.

Using the MTRNN, phases 1) and 3) are each regarded as body babbling and imitation learning, respectively. Body babbling is the process to relate the robot's actions to the outcome by repeatedly moving its body and observing the effect. Imitation learing is one of the approaches to teach a task to a robot effectively. Related works on imitation learning 
are introduced in Section II.

The result of our experiment implies the validity of our model to draw basic figures. Drawing of a circle in a clockwise direction was better performed than in an anticlockwise direction with the robot's right arm, which can also be observed with human. This could be a result of physical embodiment of the robot as the opposite phenomenon was observed with the robot's left arm.

The rest of the paper is organized as follows. Section II describes related works. Section III gives a detail of MTRNN. Section IV describes our implementation of developmental learning on drawing as preparation for the experiment. Section $\mathrm{V}$ describes the experiment setup and the experimental results. In Section VI we discuss the results of the experiment. Finally, we present conclusions and future work in Section VII.

\section{RELATED WORKS}

The function of imitation is said to be acquired during the early ages of infants for humans [4]. Imitation learning is one of the efficient means for a human to acquire new skills. Likewise, imitation learning is currently being focused as an effective method for teaching a task to robot [5].

Many approaches on robot imitation learning utilize motion capture system. Pollard et al. proposed a system to transform data acquired through motion capture with inverse kinematics to the data on robot [6]. Nakaoka et al. succeeded to imitate traditional dance using a humanoid robot with motion primitives, basic motions of a sequential motion, in addition to techniques such as motion capture and inverse kinematics [7]. The goal of these studies is mainly to reproduce a trajectory of the human motion using a robot. The drawback of these approaches is that the differences between human and robot body structure imposes a severe burden to the robot motor. Moreover, there are many parts which require manual design in the system.

Bottom-up approaches using body babbling has also been studied. Demirls et al. started imitation learning with body babbling in order to train feedback model autonomously through self-experience of interaction with environment [8]. The approach requires few preliminary manual design though the performance could not be as well as that of an approach such as motion capture system.

On imitation learning of drawing, Kulvicius et al. proposed a system to reproduce the target trajectory with high accuracy regarding both position and velocity profile using the joining method based on the modification of the original dynamic movement primitive (DMP) formulation, but the system is evaluated in simulation and they do not use a real robot [9]. Kudoh et al. succeeded in using a hand robot to draw a real object by obtaining the 3D model, extracting the characteristics using their original algorithm, and generating motion with inverse kinematics [10]. They also considered physical embodiment of the robot, but the system is designed with a top-down approach, which requires manual design. Mohan et al. utilized the primitive structures divided from an original shape with defined points called Critical Points (CP) for robot to draw a curve or a basic shape [11]. The goal was recognition of 'shapes' with the structure, but physical embodiment of robot was not considered.

These studies typically do not involve developmental factors. In other words, the self-model does not incrementally retrain during imitation learning. In this paper, we designed a developmmental imitation learning method to discuss embodiment of roobt, relative to human.

\section{Self-Model of Robot : MTRNN}

We utilize the MTRNN (Multiple Timescales Recurrent Neural Network), proposed by Tani et al. [3], as a neuro dynamical model of the robot. This model, which acts as a predictor that inputs the current state and outputs the next state, can learn and generalize multiple non-linear time sequential data. It has a hierarchical structure, comprising three neuron units: Input-Output Unit $(I O)$ for the input-output layer, FastContext Unit $(\mathrm{Cf})$, and Slow-Context Unit $(\mathrm{Cs})$ for the context layer (Fig. 1). In this study, the $I O$ consists of two neuron units, robot arm joint angles and a pen position. Each unit has a value called time constant; these values become large in the order of $I O, C f$, and $C s$. The internal state of a node that has a larger time constant is updated slower than those with small time constants. This structure provides the neuron units with various hierarchical functions. In general, the $C f$ neuron unit represents primitives of time sequential data and the $C s$ neuron unit represents the sequence of the primitives. Moreover, a specific pattern can be deterministically generated by an initial $C f$ value $\left(C f_{0}\right)$ and an initial $C s$ value $\left(C s_{0}\right)$ and parametric space of the $C f_{0}$ and $C s_{0}$ are self-organizationally acquired by the correlation between data.

We would like a robot to learn a shape as a combination of primitives in future work. Since MTRNN prossesses such capability, we utilize MTRNN as a neuro dynamical model of the robot. We can also recognize how MTRNN represents a shape by analyzing its internal representation, .

The MTRNN has three basic functions, training, recognition, and generation.

Training Weights and parametric spaces are updated with BPTT (introduced later) by inputting teaching sequential signals into $I O$.

Recognition $C f_{0}$ and $C s_{0}$ representing sequential data are calculated with BPTT by inputting the sequential data into $I O$. When the $I O$ consists of several units as in this study, recognition can be performed with at least one of the units. In this case, output errors of $I O$ that are not provided are set to zero when calculating by BPTT.

Generation The sequential data is acquired by calculating through forward calculation of RNN with $C f_{0}$ and $C s_{0}$.

Using recognition and generation, MTRNN can associate an $I O$ unit with other $I O$ unit. For example, MTRNN could recognize the pen point sequential data to generate arm joint angle time sequential data in this study. The process is done 


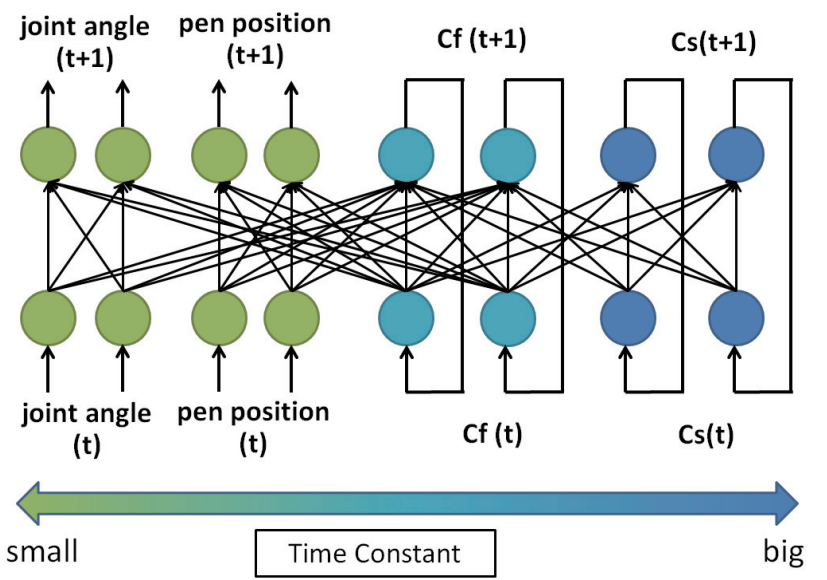

Fig. 1. Composition of MTRNN: MTRNN has three neuron unit, IO Unit (joint angle and pen position: green), Fast-Context Unit (Cf: light blue), and Slow-Context Unit (Cs: deep blue). Each neuron unit has a value called time constant, Cs with the biggest.

by first recognizing pen point sequential data to calculate $C f_{0}$ and $C s_{0}$ for generating arm joint angle. We call the function "association".

Training of the MTRNN is done using the Back Propagation Through Time (BPTT) algorithm [12]. The algorithm consists of forward calculation and weight updating.

First, the outputs of the neurons are calculated through forward calculation. The internal value of the ith neuron at step $t, u_{i, t}$ is calculated as

$$
u_{i, t}=\left\{\begin{array}{lr}
\left(1-\frac{1}{\tau_{i}}\right) u_{i, t-1}+\frac{1}{\tau_{i}}\left[\sum_{j \in N} w_{i j} x_{j, t-1}\right] & (t \neq 0) \\
0 & (t=0 \text { and } i \in I O) \\
C f_{i, 0} & (t=0 \text { and } i \in C f) \\
C s_{i, 0} & (t=0 \text { and } i \in C s)
\end{array}\right.
$$

$\tau_{i}:$ time constant of the $i$ th neuron

$x_{i, t}:$ input value at step $t$

$w_{i j}$ : weight value from the $j$ th neuron to the $i$ th neuron

$N$ : set of neurons connected to the $i$ th neuron

The output of the $i$ th neuron is calculated by applying the sigmoid function

$$
y_{i, t}=\operatorname{sigmoid}\left(u_{i, t}\right)=\frac{1}{1+\exp \left(-u_{i, t}\right)} .
$$

Using the outputs calculated in forward calculation, the weights are updated using the training error $E$ defined as

$$
E=\sum_{t} \sum_{n \in I O}\left(y_{i, t-1}-T_{i, t}\right)^{2} .
$$

The weight from the $j$ th input to the $i$ th output is updated using the derivative of the training error $\partial E / \partial w_{i j}$ as

$$
w_{i j}^{(n+1)}=w_{i j}^{(n)}-\alpha \frac{\partial E}{\partial w_{i j}} .
$$

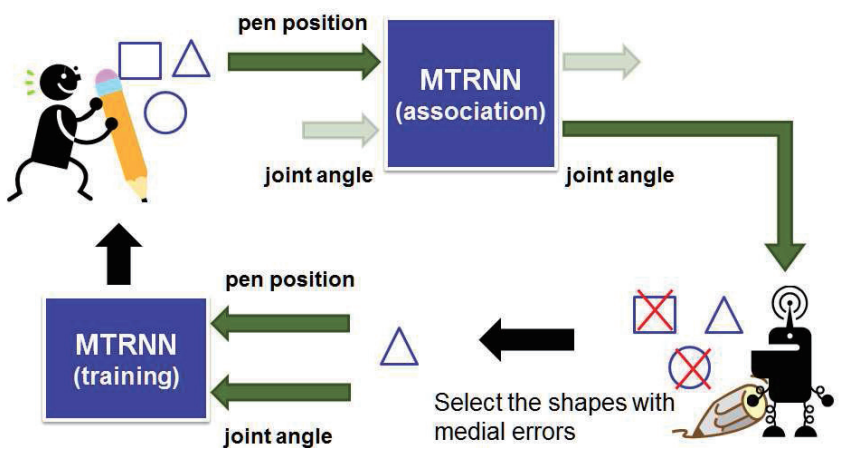

Fig. 2. Incremental imitation learning algorithm

$$
\begin{aligned}
& \alpha: \text { training coefficient } \\
& n: \text { number of updates }
\end{aligned}
$$

The $C f_{0}$ values are also updated using a back propagation algorithm along with the weight values as,

$$
C f_{i, 0}^{(n+1)}=C f_{i, 0}^{(n)}-\beta \times \frac{\partial E}{\partial C f_{i, 0}} .
$$

$$
\beta \text { : training coefficient of } C f \text { neurons }
$$

The $C s_{0}$ values are also calculated in the same manner.

\section{Process of DeVelopmental LEARning}

Our developmental learning for robot's drawing consists of two phases.

Phase1 Body babbling

Phase2 Incremental imitation learning of basic shapes

\section{A. Phase1 : Body babbling}

Phase1 is equivalent to the phase of "scribble" described in Section I. The goal of the phase is to learn the dynamics correspondence of robot's arm joint angle to that of the position of a pen point by training MTRNN with randomly generated motions.

In this phase, the robot randomly moves its arm. During the motion, sequences of robot arm joint angle and pen position are acquired. The sequences are used to train the MTRNN to associate the two sequences. Such autonomous training can reduce the cost of manually designing the robot system.

\section{B. Phase2 : Incremental imitation learning of basic shapes}

Phase2 is equivalent to the phase of "Failed Realism" described in Section I. In this phase, a robot incrementally learns basic shapes by imitating the track of shapes shown by human with the trained self-model in Phase1. Specifically, the following algorithm is repeatedly performed. The overview of the algorithm is shown in Fig. 2. 
Step1 The human draws several shapes to show to robot.

Step2 The robot recognizes the time sequential data of the shapes given in Step1 using MTRNN and estimates the initial parameters $\left(C f_{0}, C s_{0}\right)$.

Step3 MTRNN generates the time sequential data of arm joint angle with the initial parameters $\left(C f_{0}, C s_{0}\right)$ estimated in Step2.

Step4 The robot moves its arm based on the sequential arm joint angle data calculated in Step3 to draw shapes.

Step5 Calculate the squared error of the actual pen position trajectory with the predicted trajectory.

Step6 Retrain MTRNN using data with data possessing medial errors.

Step7 Go back to Step2.

Note that we cannot directly use the trajectory of shapes shown in Step1 as teaching signals for retraining as they do not include information of arm joint angle. Therefore we use the data of self-experiences given in Step4 as teaching signals for retraining. Upon selecting retraining data, we select those with medial errors, since data with small errors are needless to retrain, while those with large errors are untrainable.

\section{EXPERIMENT}

\section{A. Experiment Setup}

We experimented our proposed developmental imitation learning in drawing with the humanoid robot, NAO. NAO moved its arm using two DOF (Shoulder:Roll, Elbow:Roll) with a pen tablet as a canvas. The digital pen point position is acquired when the pen is close to the tablet. The experiment scene is shown in Fig. 3.

The size of MTRNN is four $I O$ nodes (two for arm joint angles and two for position of the pen point), $20 \mathrm{Cf}$ nodes, $8 \mathrm{Cs}$ nodes. We selected the size empirically based on the performance of body babbling training.

The specific training data used in each phase of developmental imitation learning is described below.

\section{Phase1: Body babbling}

For body babbling, random joint angles are sent to NAO to move its arm with a constant velocity. If current angles were equal to those sended, new random joint angles are sent to NAO.

NAO's joint angles and the position of the pen point of the pen tablet are acquired at $30 \mathrm{fps}$ during the babbling process. Training data set for body babbling are created by dividing the chain of motions. Note that we controlled NAO with the joint angle space not the position space. Therefore the canvas space is not equally used for drawing. The number of training data is 80 , and each data consists of 100 steps.

\section{Phase2: Incremental imitation learning of basic shapes}

In this phase, NAO learned to draw basic shapes by incremental imitation learning proposed in Sec. IV. Basic shapes include circle, triangle and square, drawn both clockwise and

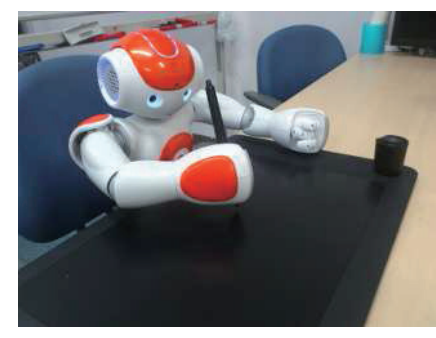

Fig. 3. Experiment Scene: The humanoid robot, NAO, is drawing with the pen tablet.

TABLE I

BASIC SHAPES TRAINED IN IMITATION LEARNING

\begin{tabular}{|c|c|c|}
\hline Circle(L) & Triangle(L) & Square(L) \\
\hline & & \\
\hline & & \\
\hline & & \\
\hline
\end{tabular}

anticlockwise from a certain point. Therefore, the number of shapes drawn in this phase is six in total. The figure of the six shapes with their labels are shown in Table I. Shapes drawn clockwise are indicated with (R) while those drawn anticlockwise are indicated with (L).

\section{B. Result with right arm}

First, we experimented with the right hand of NAO. Figure 4 shows the drawing on body babbling. As can be seen from the figure, most of the drawing consist of arcs. This is due to random control of NAO's arm using the joint angle space. This phenomenon is related to a human drawing arcs when he randomly moves his arm.

Next, Fig. 5 shows the transitions of results on incremental imitation learning. The dotted regions are due to roughness of NAO's motor often caused when moving the arm away from the robot. The results are shown for two incremental trainings since learning converged after that. We can confirm the following facts from the figure.

- Triangle(L) and Square(L) succeeded in learning. (The corners are sharply drawn.)

- Triangle(R) and Square(R) failed in learning. (The corners are round)

- Circle(R) was drawn well from the beginning.

- Circle(L) failed in learning. 


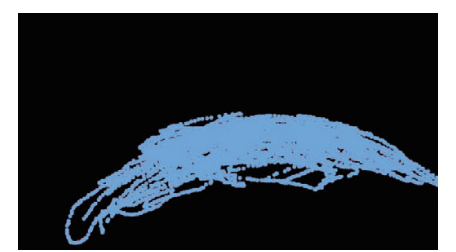

Fig. 4. Drawing of body babbling: NAO's arm is randomly controlled using the joint angle space. Therefore, most of the drawing consist of arcs.

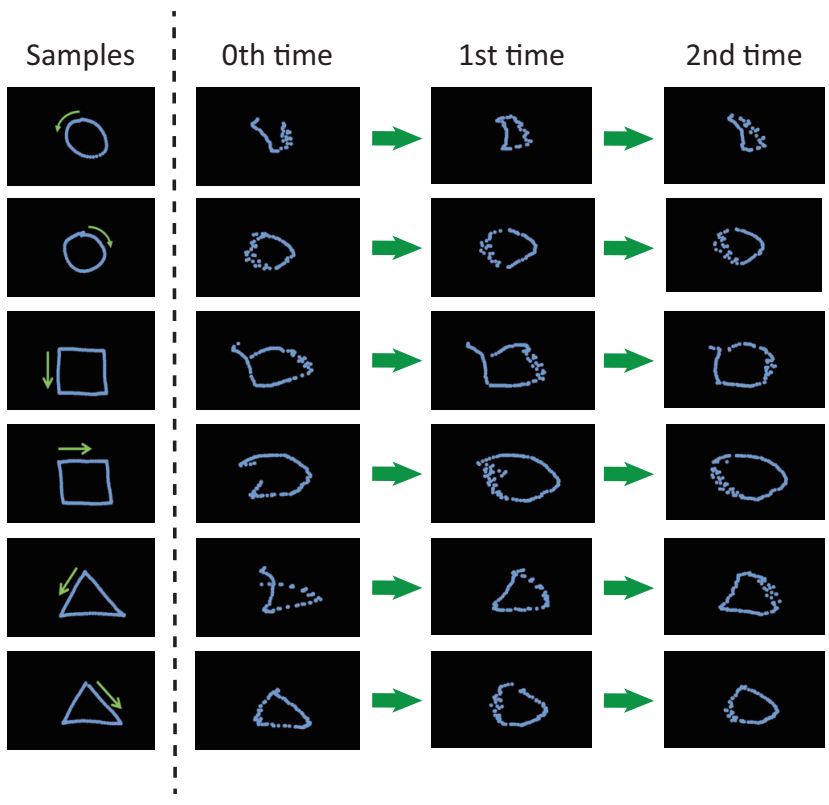

Fig. 5. Result of Incrimental Imitation Learning: The left column represents samples shown by human and the three right columns represent the transition of incremental imitation learning. It can be confirmed that results of anticlockwise square and triangle improves through the learning.

\section{Result of left arm}

To investigate the effect of body dynamics to drawing performance, we also experimented with the left arm of NAO. We expect to acquire a symmetrical result as that obtained using right arm. Figure 6 shows the result of drawing circles. Table II shows the roundness of drawing circles. Roundness is calculated as

$$
\begin{gathered}
\text { roundness }=4 \pi S / L^{2} . \\
S: \text { area } \\
L \text { : circumference }
\end{gathered}
$$

A value closer to 1 for roundness represents that the figure is closer to a circle.

As can be seen from the figure and table, NAO succeeded to draw Circle(L) and failed to draw Circle(R), which is opposite when using the right arm. The result implies that the robot tends to succeed in drawing circles when the robot starts drawing by moving its arm away from the body.

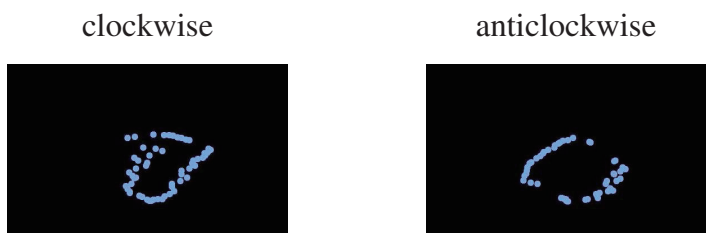

with left arm

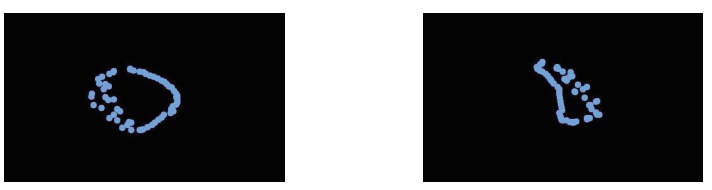

with right arm

Fig. 6. Result of drawn circles: The circles drawn with NAO's left arm and the ones drawn with NAO's right seem to be symmetric.

TABLE II

ROUNDNESS OF DRAWN CIRCLES

\begin{tabular}{|c||c|c|}
\hline & clockwise & anticlockwise \\
\hline \hline left arm & 0.617 & $\mathbf{0 . 7 0 3}$ \\
\hline right arm & $\mathbf{0 . 6 6 3}$ & 0.103 \\
\hline
\end{tabular}

\section{Analysis of Parametric Space of MTRNN}

In order to investigate the representation of shapes in MTRNN, we analyzed the parametric $\left(C s_{0}\right)$ space of the trained MTRNN. During the experiment, recognition of basic shapes using MTRNN derived $C s_{0}$ representing each shape. We then compressed $C s_{0}$ into two dimension with principal component analysis (PCA). The result is shown in Fig. 7. Shapes of each markers in the figure represent the corresponding basic shapes, and blue items represent clockwise drawing and orange items represent anticlockwise drawing.

As can be seen from the figure, clockwise and anticlockwise drawings are separated. Circle(R) and $\operatorname{Circle}(\mathrm{L})$ are located closely.

In addition to the analysis, we analysed the $C s$ space while body babbling. The right of Fig. 8 shows $C s$ space divided into grids: a color close to red represents that the segment had been used frequently during body babbling. The two figures represent the same space of Cs. Comparing the figures, it is notable that MTRNN uses the anticlockwise space more often during body babbling.

\section{DISCUSSION}

In this section, we discuss the results of the experiment.

\section{A. Embodiment and Drawing}

In the experiment, NAO succeeded in drawing clockwise circle but not an anticlockwise circle with its right arm. An opposite phenomenon was observed with the left arm. A similar phenomenon can also be seen with human. Gesell a psychologist, argues that infant's drawing of a circle is more circumscribed than at 3years, and charactersistically 


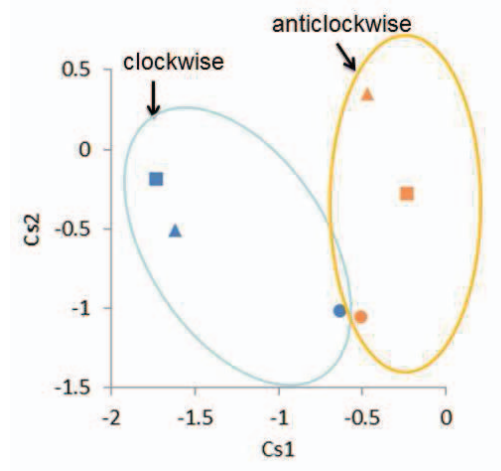

Fig. 7. Representation of $C s_{0}: C s_{0}$ of each figure acquired through training are plotted. Shapes of each markers represent the corresponding basic shapes. It can be confirmed that clockwise and anticlockwise drawings are separated.
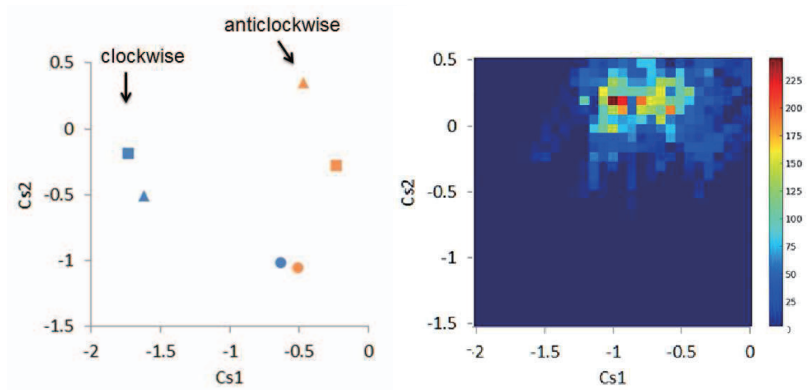

Fig. 8. Analysis of $C s$ space: The left is same as Fig. 7 and the right shows $C s$ space divided into grids: a color close to red represents that the segment had been used frequently during body babbling. Comparing the figures, it is notable thet MTRNN uses the anticlockwise space more often during body babbling.

is executed in a clockwise direction, appropriate to a more strongly entrenched right-handness [13]. In addition, drawing a circle is not much influenced by body babbling since $C s$ of the circles are not within the area of $C s$ used in babbling as can be seen from Fig. 8. This result implies that the body structure is suited for drawing circles as arcs were frequently drawn during babbling.

\section{B. Babbling Experience and Drawing}

In the experiment, the triangle and square drawn in an anticlockwise direction were better performed than those drawn in a clockwise direction. As described in Section V-D, the anticlockwise space of $C s$ is used more during body babbling. Since our learning is based on body babbling, NAO naturally learned to draw better in the direction that it has experienced more.

\section{CONCLUSION}

This paper mainly dealt with robot developmental learning on drawing and discussed the effect of physical embodiment to the task. It is valuable for creating a consistently developing robot and feedback to other fields such as cognitive science or developmental science.

In this study, we designed developmental learning on drawing refering to human development. First, we utilized MTRNN for the robot's dynamics model in order to learn drawing as dynamics. Second, we configured developmental learning with two phases: the first phase is body babbling (Scribbling) and the second phase is imitation learning (Failed Realism).

Our experiment results and analysis confirm that drawing of circles is related to physical embodiment, while square and triangle were learned through the babbling process. A similar phenomenon could be observed in human development.

As our next step, we plan to implement the second phase of development on human drawing, "Fortuitous Realism", into our developmental learning. In this phase, an infant incidentally discovers the similarity between a figure and an object, and draws the object he looks at. By adding the phase, the robot would select specific figures during body babbling and learn them intensively. We expect that our approach will contribute to discover valuable and interesting facts about physical embodiment related to drawing.

\section{ACKNOWLEDGMENT}

The work has been supported by JST PRESTO "Information Environment and Humans", MEXT Grant-in-Aid for Scientific Research on Innovative Areas "Constructive Developmental Science" (24119003), Grant-in-Aid for Young Scientists (B) (25730519), Kayamori Foundation of Informational Science Advancement, and Tateishi Science and Technology Foundation.

\section{REFERENCES}

[1] M. Asada, K. MacDorman, H. Ishiguro, and Y. Kuniyoshi: "Cognitive developmental robotics as a new paradigm for the design of humanoid robots," Robotics and Autonomous Systems, vol. 37, pp. 185-193, 2001.

[2] G. H. Louquet: "Le Dessin Enfantin," 1927.

[3] Y. Yamashita and J. Tani: "Emergence of Functional Hierarchy in a Multiple Timescale Neural Network Model: a Humanoid Robot Experiment," PLoS Comput. Biol., Vol. 4, No. 11, e1000220, 2008.

[4] A. N. Meltzoff and M. K. Moore: "Imitation of facial and manual gestures by human neonates," Science, Vol. 198, pp. 75-78, 1977.

[5] S. Schaal: "Is Imitation Learning the Route to Humanoid Robots?," Trends in Cognitive Sciences, Vol. 3, No. 6, pp. 233-242, 1999.

[6] N. S. Pollard, J. K. Hodgins, M. J. Riley, and C. G. Atkeson: "Adapting Human Motion for the Control of a Humanoid Robot," Proceedings of the IEEE International Conference on Robotics and Automation, pp. 1390-1397, 2002.

[7] S. Nakaoka, A. Nakazawa, K. Yokoi, H. Hirukawa, and K. Ikeuchi: "Generating Whole Body Motions for a Biped Humanoid Robot from Captured Human Dances," Proc.of 2003 IEEE International Conference on Robotics and Automation, pp. 3905-3910, 2003.

[8] Y. Demirls and A. Dearden: "From motor babbling to hierarchical learning by imitation: a robot developmental pathway," Proceedings of the 5th International Workshop on Epigenetic Robotics, pp. 31-37, 2005.

[9] Kulvicius T., Ning, K., Tamosiunaite M., and Worgotter F.: "Joining Movement Sequences: Modified Dynamic Movement Primitives for Robotics Applications Exemplified on Handwriting," Robotics, IEEE Transactions on, Vol. 28, pp. 145-157, 2012.

[10] S. Kudoh, K. Ogawara, M. Ruchanurucks, and K. Ikeuchi: " Painting robot with multi-fingered hands and stereo vision," Robotics and Autonomous Systems, Vol. 57, No. 3, pp. 279-288, 2009.

[11] V. Mohan, P. Morasso, G. Metta, and S. Kasderidis: "Teaching a humanoid robot to draw 'Shapes," Autonomous Robots, 31(1), pp. 21-53, 2011.

[12] P. Werbos: "Backpropagation through time: What it does and how to do it," Proceedings of the IEEE, Vol.78, No.10, pp.1550-1560, 2002.

[13] A. Gesell: "The First Five Years of Life: a guide to the study of the preschool child," Harper \& Brothers, Sec. IV, Part D, 1940. 